\title{
Emergency Response Planning and Training through Interactive Simulation and Visualization with Decision Support
}

\author{
Bruce Donald Campbell, Huseyin Onur Mete, Tom Furness, Suzanne Weghorst, Zelda Zabinsky \\ Pacific Rim Visualization and Analytics Center (PARVAC) \\ University of Washington, Seattle, Washington \\ brucedc, mete, tfurness, weghorst, zelda@u.washington.edu
}

\begin{abstract}
Teams of first responders work together to effectively manage a community-wide crisis. Traditionally, key groups such as police, fire, and medical are each trained for specific emergency procedures. Emergency response teams design training exercises to augment an individual's cognition associated with performing time critical roles. The aggregation of all individual cognition, distributed through communications, suggests a situation awareness that an incident commander requires to perform optimal decision-making. We are developing a computer-supported simulation environment with a decision support tool called the RimSim, to facilitate emergency response planning and training of first responders. We modularize RimSim for synchronous multi-player use or asynchronous individual use with simulated participants. Through interactive computersupported role-play with shared visualizations, we are able to study distributed cognition with a long-term goal of identifying opportunities for improving information management during emergency response. We aim to improve mindful distributed cognition for first responders during emergency response to natural and man-made crises.
\end{abstract}

\section{INTRODUCTION}

First responders deserve ample opportunity to plan and train for their contribution within community-wide crisis response scenarios. We have observed some shortcomings of requiring synchronous participation in drills and tabletop exercises. Personnel who manage unforeseen crises on a daily basis fill Emergency Response Center (ERC) roles. Synchronous participation can be augmented by asynchronous opportunities to train. Computer-based simulations offer the ability to participate in asynchronous training exercises. The RimSim simulation environment enables first responders to familiarize themselves with other roles within a response effort in order to improve situation awareness and distributed cognition. Through repeat dealings with complex decision-making response scenarios, first responders can become skilled in training their readiness by learning how to be more mindful about how they think as well as gaining the ability to create a thought process that leads to better perceptual and cognitive flow during stressful times.

Decisions to support preparedness and response activities for disaster management are challenging due to the uncertainties of events and complications due to the lack of real time data. Current societal plans for first response preparedness include appropriate division of labor for first responders and emergency operation center personnel through guidelines like the National Incident Management System (NIMS) and a slowly improving public awareness of appropriate response actions by the general public. Across the country, emergency response drills and tabletop exercises are taking place to familiarize role players with their duties in emergency response scenarios.
Within the Regional Visualization and Analytics Center (RVAC) community, the Purdue University RVAC (PURVAC) [1] is involved with simulating large response activities with the Indiana Department of Homeland Security in their Muscatatuck urban training facility [2]; the University of Washington Health Center (UWHC) emergency response teams stage regular drills to prepare hospital staff for a wide variety of emergency scenarios; and the Northeast Visualization and Analytics Center (NEVAC) at Penn State University continues to develop a NeoCITIES program to test team collaborative decision-making processes in a command and control environment [3]. Drills and exercises typically take place every three to six months and test a different emergency response scenario each time.

Large community disaster events like Katrina and September 11 are not reoccurring events with which communities can train physically for emergency response. As a result, we look to create planning and training opportunities through other means that can provide an appropriate mental model for visualizing and simulating effective action. Paper-based workshops are useful for flushing out an overall plan and its effectiveness, but individuals may wish to train repetitively with multiple scenarios to develop a strong sense of their potential contribution under varying conditions, especially if they want to become more mindful of their thought process. The range of potential man-made and natural emergency scenarios continues to increase as transportation and information networks expand worldwide.

The RimSim team has developed a modular software development model with which to build emergency response simulations and has begun to build a scenario for five members of a medical logistics team within an urban center ERC. To test our methodology, we consider two major earthquake scenarios within the Seattle, Washington area: Seattle Fault and Cascadia Subduction Zone. We acknowledge the difficulty of building a realistic scenario of such magnitude, but believe we can focus on representative complexity and data sharing overlap to the point of building an asynchronous planning and training tool for first responder mindfulness.

\section{MOTIVATION}

\section{The Embodied Mind}

A growing community of cognitive scientists test hypotheses that our minds develop primarily through interaction with the external world. We grow our minds through experiences and our cognition becomes the culmination of the history of our experiences. We learn through interaction with objects and use our bodies often to do so. Francisco Varela et al. refer to the embodied mind that exists when the mind is brought closer to the body as opposed to becoming disembodied through abstract, openended reflection [4]. Edwin Hutchins' ethnographic studies of large ship navigation teams demonstrated how 
sophisticated cognition could arise through people interacting with external objects, shared artifacts, and other people without requiring abstract internal representations [5]. Hutchins details how such a distributed cognition makes a team of navigators ready for emergency response scenarios encountered during navigation in narrow waters with changing environmental conditions.

Emergency response drills allow teams of emergency response workers in an ERC to work together to practice their roles in the chaotic environment they will likely encounter at crisis time. Drill participants gain practice using the external tools with which they expect to be equipped. They gain practice communicating through shared artifacts they expect to generate for emergency management. They gain practice communicating and problem solving with other team members and across ERC teams. These drills seem the more valuable when embracing the hypothesis of an embodied mind as first responders embody tools, artifacts, and people as extensions of their mind-body resource. Each participant has the opportunity to perform their role and drill coordinators can make sure the whole ERC is structured as effectively as possible. As a collaborative unit, they aim to share one superset embodied mind.

Drill participants are not always provided details about the embodied mind hypothesis before they participate in a drill. This makes sense when the coordination team is watching the drill and changing conditions to keep the drill participants active. If a person or team is done managing an issue that arises, coordinators inject another to complicate roles so participants must remain engaged. When performed in this manner, drills push the cognitive load for each team member such that they have little slack for considering how they are thinking about the problem - the meta-thinking Richard Heuer Jr. suggests is significant to develop for gaining maximum effectiveness [6].

Evaluating the success of a drill across all participants is a tedious and demanding process for the drill coordination team. Evaluators focus on evaluating the distribution of labor, roles, and verification that the physical tools, artifacts, and social relationships shared among the ERC and first responders in the field are well designed for the scenario tested. Given the high cost of such evaluations, additional testing for the embodied mind hypothesis during drill time taxes the overall system evaluation.

The RimSim provides individual training opportunities to allow a participant to consider the embodied mind perspective for learning. As we consider the mind as an extension of the body, we see opportunities to train the mind in ways congruent to how we train the body. When training the body for a task, we explicitly identify the steps we want to take to perform a complex task. When performing emergency response, we can identify the thought process we wish to take to perform a complex task as well. These steps include manipulating external tools, interacting with shared artifacts, and communicating with other people. By externalizing our thought process, we can train our mind similarly to how we train our body - a potential benefit of considering and testing the embodied mind hypothesis.

As we consider the embodied mind hypothesis, we find participants who are mindful of the thought actions they are taking have a better chance of avoiding error. Mindfulness comes through experience that requires repetition to train the mind - in a manner similar to how a musician practices playing music with her instrument. Repeat role-play under changing conditions and replay of same conditions allows a participant to develop mindfulness as a skill that can be applied to any crisis, training the mind as a firefighter trains his body to manage heavy equipment effectively under stress. To maximize simulated role-play opportunities the RimSim architecture supports both synchronous and asynchronous training modes.

\section{Synchronous or Asynchronous Training}

Repetitive role-play within a scenario builds experience in dealing with complexity under urgent conditions. In a live drill, participants cannot realistically stop the drill to identify a mistake they made and roll back the drill to correct the error and continue forward. Significant enough errors more often require a new drill following a debriefing that occurs after the fact. Participants learn the significance of making errors in a realistic way because mistakes aren't easily corrected in a live crisis, but they might never get a chance to play out that scenario with what they think is the right action. Validating error hypotheses becomes difficult and corrective actions are not re-enforced. The ability to train asynchronously provides a participant more flexibility in adjusting for errors through individual time lines.

In a team environment, many errors are the result of communication breakdowns that are harder to simulate due to the wide variety of communication styles and communication devices. Where team members need to communicate often, synchronous role-playing seems necessary for training the social cognition aspects of an embodied mind approach. Seeing benefits to both asynchronous and synchronous training through simulation, RimSim architecture supports both on a role-by-role basis.

\section{RIMSIM ARCHITECTURE}

We have modularized the software support facilities of RimSim in order to allow parallel development of code bases needed to implement software-based simulation. We believe an iterative development model allows us to test out useful ideas rapidly and get immediate feedback. Our RimSim architecture in Figure 1 supports the implemented simulation shown in Figure 2.

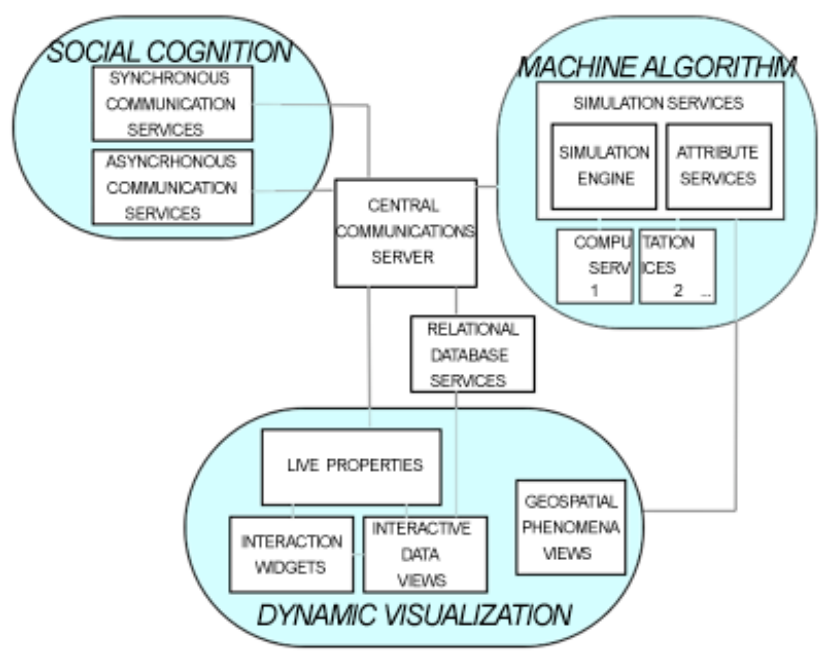

Figure 1. 'Ihe RimSim Architecture. 
We engineer RimSim components to interact well in three module clusters to support Stuart Card's recommended model of technology support for augmenting cognition in complex activities [7]. Card's model identifies three major categories for cognition support components: social cognition modules supporting asynchronous and synchronous communications services between first responders and the ERC are iterated upon to reflect realistic communications channel behavior during emergencies; dynamic visualization modules are provided for shared artifact investigation using shared geospatial visualization with dynamic layers that build and communicate situation awareness over time; machine algorithm support features are provided primarily through a simulation engine that manages simulation attributes for sharing among agents in the simulation. We develop and add other algorithm support modules to communicate through the simulation engine services interface.

Modular software provides an opportunity for flexibly designing individual and group emergency response simulation exercises. We iteratively build modules to encode our framework in RimSim software that individuals can use to plan or train on scenarios with other live participants or with simulated participants. By remaining faithful to the master RimSim architecture plan, we assure our modules some reusability and interoperability for future scenario development.

We design communications server architecture to enable multiple live participants to pass messages between instantiations of any RimSim client. The same communication server also enables messages to be passed between simulated role-players to live players to the point where only one live player need be planning or training at any point in time. Synchronous communication channels provide text and voice communications between live participants that can be degraded to reflect inferior communication channels so often encountered in crisis scenarios. Asynchronous communication channels allow first responders in the field to upload text, images, video or global-positioning information without requiring a synchronous communicator at the other end of the communication.

To support asynchronous training, RimSim simulation services must support simulated participants similar to how other key agents in a role-play scenario are simulated. Agent-based frameworks have been developed and validated to support a wide range of simulation subjects [8]. Time, cost, material usage, and human health metrics help a first responder assess their aptitude in performing first responder roles, but to do so all relevant entities existing in an emergency response scenario must be simulated. Simulating another role-player represents the complex end of the simulation challenge, but existing general-purpose, multiple, agent-based frameworks are appropriate for simulating many emergency response activities, especially those associated with logistics roles identified in the NIMS Handbook. We architect RimSim to be able to incorporate an agent-based framework for simulation support.

Many machine algorithm tools are helpful in supporting emergency response. We see a historical transition of reliance upon external mechanical devices towards more and more reliance upon electrical computing devices in emergency response. Although first responders have a reduced capacity for performing mental calculations under time pressure duress, they become very capable at incorporating the output of complex computations if presented in a way consistent with traditional heuristics first responders have been trained upon.

Mechanical devices are less dependent on communications channels and provide a more literal use of the embodied mind through direct manipulation of the body, but we believe computer-driven computation services will continue to become more and more popular as decision-support tools. The RimSim architecture allows for decision-support modules to participate with core simulation services for role-playing scenarios such that first responders can manipulate decision support tools with an embodied mind.

As relevant role-play data is shared between live roleplayers, simulated role-players and decision-support services, dynamic visualization services provide shared artifacts role-players can use to plan and train their actions As both the scientific visualization and information visualization communities test out an increasing number of visualization techniques for human sense-making and situation awareness activities, we design the RimSim architecture to incorporate solutions that show promise for the role-play scenarios we wish to train. Whole improvisional visualization frameworks like Prefuse [10] or Improvise [11] can be incorporated into a RimSim client or handcrafted solutions can be tried on the fly as well. A relational data services component mediates data organization between the simulation services and the dynamic visualization services.

\section{AN IMPLEMENTATION FOR EMERGENCY RESPONSE}

The RimSim team attended a variety of emergency response drills at ERCs of different sizes: county, city, and neighborhood. We found a response team of five medical logistics personnel who worked at the city level to improve medical supply and personnel first response to an injured public. In combating a major chlorine gas leak scenario, the medical logistics team used physical tools, external artifacts, and social relationships within the team and with other ERC teams in a manner that suggested good distributed cognition as defined by Hutchins [5]. We began to create a simulation framework for use in training a medical logistics team for emergency response during earthquakes.

\section{Medical Logistics Case Study}

Upon interviewing and observing medical logistic team responders in action within the ERC at the University of Washington Medical Center, the RimSim team modelled the medical logistics team as performing five critical activities: coordinating supplies received from other jurisdictions, realtime allocation of supplies to point-of-use, bed management for injured persons across all resources, generation of makeshift shelters and new points of service, and traffic management for supply delivery trucks and medical response vehicles. Medical logistics team members share a situation awareness that grows in importance whenever decision-making for one activity overlaps with decisionmaking for another. Team members share geospatial and temporal data needs regarding medical casualties, vehicle routing, first responder locations, point-of-service locations, medical supply inventories, and other resources. 
We offer RimSim architecture to support an iterative, modular software development process that lets us test out useful response plans rapidly to get timely user feedback. We studied an earthquake scenario within the medical logistics team and implemented a first aid response simulator for the real-time allocation of supplies to point-ofuse activity logician to train upon. As a key activity within the earthquake scenario, we developed a decision support module to assist with the decisions regarding the location and allocation of medical supplies. For disaster response, the optimization model provides support for the transportation of materials from warehouses to hospitals, including the loading and routing of vehicles. As a result, the medical logistics team has access to a decision-support system while playing the RimSim. We iterate upon the RimSim display to improve activity cognition through a shared dynamic visualization.

\section{Dynamic Visualization}

We iterate upon the RimSim display to improve cognition through visualizations that support an externalized thought process of an embodied mind. Figure 2 shows a RimSim architecture supported role-play client display in action. Using the display to support embodied thinking, the role-player, interacting with an unfolding emergency response crisis scenario simulation, tries to minimize costs while minimizing loss of life.

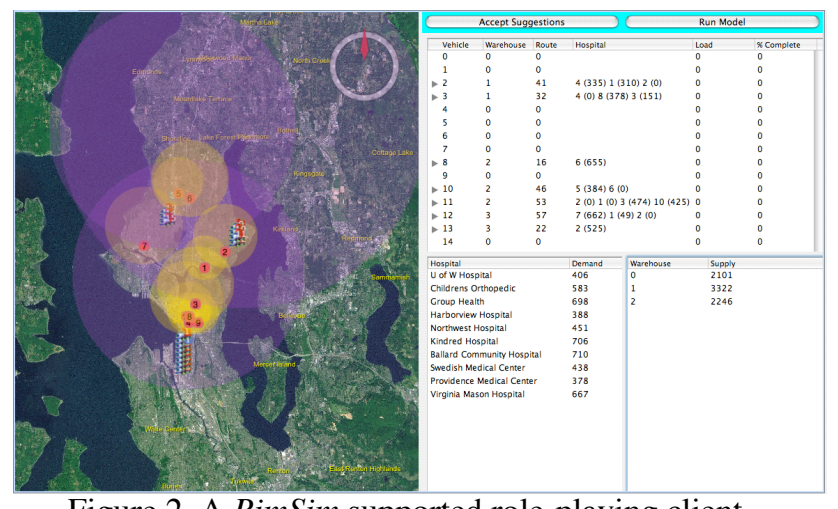

Figure 2. A RimSim supported role-playing client

To demonstrate the flexibility of our architecture, we plugged in a whole-Earth globe visualization service for our dynamic visualization component and built a decisionsupport tool to verify our architecture could communicate with simulation services to support role-play training in real-time. As a coordinating artifact, familiarity with a consistent geospatial visualization assists in knowledge construction and decision support [9]. The RimSim software application in Figure 2 is representative of the interface and dynamic visualization that all role players could use during a distributed response - consisting of a geospatial interface with a drill-down traffic management system, and animation of the simulated actual or predicted movement of critical resources. Tabular resource management widgets provide redundant information for those who lack map interpretation skills.

The fact role-players use the visualization and support services in Figure 2 to embody resource sinks and sources as objects in their mind verified our overall architecture as relevant to training an embodied mind. A central communications server shares simulation and visualization data between actual or simulated roleplayers. A relational data service keeps track of local attribute values within each role-player's application client. We iterate upon the RimSim display to improve cognition through visualization of all relevant attributes, allowing us to demonstrate our ideas while continually improving each component independently.

We continue to validate our RimSim architecture through the development of future dynamic visualization modules in a similar manner. As we talk to users, we hear concerns about how clutter in the display contributes to information overload. Role-players want information presented in ways they are familiar with in order to better apply an embodied mind to the recognition-primed decision making process that Gary Klein popularizes through his research [12]. To reduce cognitive load, we implemented an example of a machine algorithm service to support embodied thinking through an advanced heuristic computation that could not reasonably be calculated in real-time by the role player.

\section{Optimization for Decision Support}

The location and inventory levels of stored medical supplies must be shown to have a low risk of earthquake damage themselves, yet provide fast distribution to hazardous areas. Moreover, the transportation routes are at risk of sustaining damage, which has to be considered in the allocation of materials to point-of-use. Mete and Zabinsky [13][14] present optimization models for disaster preparedness and response to assist with decisions for the location and allocation of medical supplies to be used in emergencies in the Seattle area that is vulnerable to earthquakes. Hospitals in the area share nine warehouses for medical supply storage and experts predict 22 hospitals, in a major earthquake scenario, will have higher demand than their daily operations. Considering the possible disaster scenarios for the Seattle area, their stochastic programming model generates an optimal plan for the location and inventory levels of medical supplies in the preparation phase and an initial plan for the transportation of medical supplies in earthquake response.

Upon simulating the onset of disaster scenarios, RimSim provides continuous monitoring of the disaster zone, which is necessitated by the dynamic nature of the earthquake response, and enable a role-player to conduct medical supply distribution according to overall disaster field information. As well as the demand of hospitals, we monitor the supply amounts in warehouses including the incoming aid contributions from external jurisdictions. The current road availabilities and vehicle assignments are also presented. RimSim role-players can either suggest their own vehicle assignments and routing or consult with the embedded mixed-integer programming model to accept suggestions provided by Mete and Zabinsky [13][14] for optimal loading and routing of vehicles. The role-player has liberty to apply the solution partially, send off their own supply truck decisions, and re-run the model to see any new suggestions for supply deliveries based on the resultant state. The decision support module can be called repeatedly as the field information continues to arrive.

Even though the focus of the mathematical model has been on decision support for emergency response scenarios, we have found the work to be seamlessly implemented within a larger planning and training simulation that need not focus on decision support, but role-play experience for developing the embedded mind of the player. Given the modular and iterative development approach to testing out the RimSim architecture, other heuristic support modules can be 
provided for complex problem solving support in a similar manner.

\section{CONCLUSION}

As an emergency response-planning tool, geospatial visualizations promote distributed cognition through shared artifacts. The visualizations are only as good as the data contained within and the tool is only as good as its usability. Through identifying mental models that support an embodied mind hypothesis and creating a flexible RimSim architecture to advise role-play application development for role-play training and planning, we work to improve first responder participation in data collection and first response activity with software that presents a useful complexity level of crisis scenarios for emergency response planning and training.

Upon building RimSim architecture to align with popular models of augmenting cognition through technology, we tested out our architecture through development of a roleplay training solution for a member of the medical logistics team within an ERC. As RimSim is an approach more than a specific solution, we continue to search out collaborative opportunities for incorporating other software modules into RimSim implementations. Through continued use of RimSim supported role-play simulation opportunities, we aim to generate useful discussion on the applicability of the embodied mind hypothesis in supporting individual and distributed cognition among first responders for effective performance. We hope a major by-product of betterdistributed cognition will be a better situation awareness that helps all participants in emergency management.

\section{ACKNOWLEDGMENTS}

We thank the Pacific Rim Visualization and Analysis Center (PARVAC), the National Visualization and Analytics Center (NVAC), and the Department of Homeland Security (DHS) for providing primary funding and support for the RimSim project.

\section{REFERENCES}

[1] PURVAC: Purdue University Regional Visualization and Analytics Center, http://www.purvac.org/ (Accessed Mar 1, 2008).

[2] Muscatatuck Urban Training Center, http://www. mutc.org/ (Accessed Mar 1, 2008).

[3] McNeese, M. D., Bains, P., Brewer, I., Brown, C. E., Connors, E. S., Jefferson, T., Jones, R. E., \& Terrell, I. S., "The NeoCITIES Simulation: Understanding the design and methodology used in a team emergency management simulation," Proceedings of the Human Factors and Ergonomics Society 49th Annual Meeting (pp. 591-594), Santa Monica, CA, 2005.

[4] Varela, F., Thompson, E., Rosch, E., The Embodied Mind: Cognitive Science and Human Experience, Cambridge, MA: MIT Press, 1991.

[5] Hutchins, E., Cognition in the Wild, Cambridge, MA: MIT Press, 1995.

[6] Heuer Jr., R. J., Psychology of Intelligence Analysis, Washington, DC: CIA Press, 1999.
[7] Card, S., "Technology Support for Augmented Cognition," Speech at the Information Visualization Award Acceptance Presentation, Sacramento, CA: VIS Conference, 2007.

[8] Rojas, E. and Mukherjee A., "A General Purpose Situational Simulation Environment for Construction Education," Journal of Construction Engineering and Management, ASCE, 131(3), 2005.

[9] Adams, M.J., Tenney, Y.J., and Pew, R.W., "Situation Awareness and Cognitive Management of Complex Systems," Human Factors Special Issue, 37(1):85-104, 2001.

[10] Heer, J., Card, S., and Landay, J., "Prefuse: a toolkit for interactive information visualization." In CHI 2005, Human Factors in Computing Systems, 2005.

[11] Weaver, Chris, "Building Highly-Coordinated Visualizations In Improvise”, Proceedings of the IEEE Symposium on Information Visualization 2004, Austin, TX, 2004.

[12] Klein, Gary, Sources of Power: How People Make Decisions, Cambridge, MA: MIT Press, 1999.

[13] Mete, O. N., Zabinsky, Z., "Stochastic Optimization of Medical Supply Distribution," Poster Presentation, Harvey Mudd College Mathematics Conference on Public Sector Operations Research, September 28-29, 2007.

[14] Mete, O. N., Zabinsky, Z., "Stochastic Optimization of Medical Supply Distribution in Disaster Management", Seattle, WA: Informs Annual Meeting Proceedings, November 4-7, 2007. 